\title{
As causas locais de superlotação em um serviço hospitalar de emergência de Sergipe
}

\author{
Local causes of overcrowding in an emergency hospital in Sergipe \\ Causas locales del hacinamiento en un hospital de urgencias de Sergipe
}

Recebido: 07/11/2021 | Revisado: 11/11/2021 | Aceito: 11/11/2021 | Publicado: 15/11/2021

\author{
Romário Torres Alcântara \\ ORCID: https://orcid.org/0000-0003-2393-8061 \\ Universidade Tiradentes, Brasil \\ E-mail: romalcmed@gmail.com \\ André Luiz Baião Campos \\ ORCID: https://orcid.org/0000-0002-5794-7196 \\ Universidade Tiradentes, Brasil \\ E-mail: andre.baiao@souunit.com.br \\ Jessika Valeska Martins Ramos \\ ORCID: https://orcid.org/0000-0001-7449-3694 \\ Universidade Federal de Sergipe, Brasil \\ E-mail: jessikaramosenfa@hotmail.com
}

\begin{abstract}
Resumo
A superlotação é um problema presente mundialmente que assola os mais diversos países e serviços públicos de saúde, assim, a identificação de suas causas é um desafio para gestores e líderes governamentais. Diante disto, este estudo objetivou descrever as principais causas locais de superlotação em um serviço de emergência do estado de Sergipe. Alguns dos passos galgados para chegar lá foram conceituar superlotação em serviços hospitalares de emergência e apresentar as principais causas de superlotação, descrever as características do serviço estudado bem como apontar seus dados estatísticos de superlotação, comparar estes dados com as causas apresentadas e diante disso a possível causa para a superlotação. Para tanto, foi utilizado como método para coleta de dados a pesquisa documental, através do estudo de dados estatísticos frente a referenciais teóricos acerca da Superlotação de Serviços Hospitalares de Emergência e suas causas e dados publicados pelo DATASUS. A partir da análise dos dados foi possível identificar alguns fatores causais desencadeadores da superlotação no serviço estudado. Observou-se um grande influxo de pacientes ao serviço, bem como uma média de permanência elevada devido à escassez de recursos para atender a demanda do serviço. Enfim, por meio de todo o estudo realizado e dos dados apresentados foi possível se identificar que a superlotação no serviço estudado possui causas multifatoriais e há a necessidade de uma maior investigação. Desta forma, faz-se necessário o incentivo a mais pesquisas e ao aprimoramento da disponibilidade de dados para análise.
\end{abstract}

Palavras-chave: Serviço hospitalar de emergência; Aglomeração.

\begin{abstract}
Overcrowding is a globally present problem that plagues the most diverse countries and public health services, thus, identifying its causes is a challenge for managers and government leaders. Therefore, this study aimed to describe the main local causes of overcrowding in an emergency service in the state of Sergipe. Some of the steps taken to get there were conceptualizing overcrowding in emergency hospital services and presenting the main causes of overcrowding, describing the characteristics of the service studied as well as pointing out its statistical overcrowding data, comparing these data with the causes presented and, therefore, the possible cause for overcrowding. For this purpose, documentary research was used as a method for data collection, through the study of statistical data against theoretical references about the overcrowding of Emergency Hospital Services and its causes and data published for DataSus. From the data analysis, it was possible to identify some causal factors that trigger overcrowding in the studied service. There was a large influx of patients to the service, as well as a high average length of stay due to the scarcity of resources to meet the service's demand. Finally, through the entire study carried out and the data presented, it was possible to identify that overcrowding in the service studied has multifactorial causes and there is a need for further investigation. Thus, it is necessary to encourage more research and improve the availability of data for analysis.
\end{abstract}

Keywords: Emergency service, Hospital; Crowding.

\section{Resumen}

El hacinamiento es un problema presente a nivel mundial que afecta a los más diversos países y servicios de salud pública, por lo que identificar sus causas es un desafío para gerentes y líderes gubernamentales. Por tanto, este estudio 
tuvo como objetivo describir las principales causas locales de hacinamiento en un servicio de emergencia en el estado de Sergipe. Algunos de los pasos que se dieron para llegar allí fueron conceptualizar el hacinamiento en los servicios de urgencias hospitalarias y presentar las principales causas del hacinamiento, describir las características del servicio estudiado, así como señalar sus datos estadísticos de hacinamiento, comparar estos datos con las causas presentadas y, por tanto, , la posible causa del hacinamiento. Por tanto, se utilizó la investigación documental como método de recogida de datos, mediante el estudio de datos estadísticos contra marcos teóricos sobre el hacinamiento de los Servicios de Urgencias Hospitalarias y sus causas y datos publicados por DATASUS. A partir del análisis de datos, fue posible identificar algunos factores causales que desencadenan el hacinamiento en el servicio estudiado. Hubo una gran afluencia de pacientes al servicio, así como una estadía promedio alta debido a la escasez de recursos para atender la demanda del servicio. Finalmente, a través de todo el estudio realizado y los datos presentados, se pudo identificar que el hacinamiento en el servicio estudiado tiene causas multifactoriales y existe la necesidad de una mayor investigación. Por tanto, es necesario fomentar más investigaciones y mejorar la disponibilidad de datos para el análisis.

Palabras clave: Servicio de urgencia en hospital; Aglomeración.

\section{Introdução}

O serviço hospitalar de emergência pode ser conceituado como sendo um local que possui os mais diversos recursos de saúde para se prestar um atendimento de emergência e/ou urgência à população. Desta forma, a superlotação de tal serviço se trata de um problema que é caracterizado quando a quantidade de pacientes em atendimento excede a capacidade local do serviço.

De forma geral, a superlotação de um serviço hospitalar de emergência deve ser investigada para buscar intervenções que visem solucionar o problema. Com isso, uma análise global do serviço para se identificar as principais causas que possam justificar a situação deve ser realizada e a partir dela se traçar planos de solução.

O fato de a superlotação de serviços de emergência ser um problema presente mundialmente e trazer os mais variados prejuízos ao serviço de saúde, provoca os gestores em saúde a buscarem estratégias e intervenções para este problema. Uma das formas de traçar estratégias é realizando análises de cenário e buscar os fatores causais que desencadearam a situação problemática.

Um dos primeiros passos para compreender melhor a superlotação hospitalar de serviço de emergência, é a identificação em estudos científicos de alguns fatores causais para a superlotação. Esta, por vezes está associada a problemas que permeiam o processo de atendimento dos pacientes no sistema de saúde. A entrada, permanência e saída dos pacientes no serviço, são etapas deste processo que quando expostas mostram que suas alterações influenciam direta e/ou indiretamente a ocorrência de superlotação.

Após análise do consenso científico sobre o assunto, é necessária uma análise do serviço em si como um todo, descrever as características do serviço de emergência em questão, verificar os dados estatísticos de domínio público, e em seguida realizar uma comparação dos dados apontados frente as características do serviço. A junção desses dados fará ser possível levantar principais causas de superlotação e desta forma apresentar a possível causa para a superlotação do serviço de emergência estudado.

Diante do exposto acima, este estudo buscou reunir dados/informações com o propósito de responder ao seguinte problema de pesquisa: Quais as principais causas locais de superlotação em um serviço de emergência do estado de Sergipe?

O objetivo geral do estudo, visa descrever as principais causas de superlotação hospitalar de um serviço de emergência do estado de Sergipe e permitir que intervenções possam ser geradas visando uma melhor qualidade do serviço.

Para alcançar este objetivo, esta pesquisa reuniu dados de pesquisa bibliográfica e dados estatísticos de domínio público, para análise documental, a fim de explorar e descrever o cenário e elencar as causas locais de superlotação. 


\section{Metodologia}

Como bem nos assegura Lakatos \& Marconi (2021), pode-se dizer que pesquisa é um processo para investigação de fenômenos e ampliação do conhecimento que leva a reflexão. Neste contexto, fica claro que permite analisar e investigar a realidade com objetivo de novos conhecimentos. O mais preocupante, contudo, é constatar que sua composição pode ser difícil e demandar esforço.

“A Pesquisa Básica, também denominada Pesquisa Pura o Teórica, é aquela que amplia o conhecimento científico a partir de novas articulações teóricas e proposições conceituais” (Sakamoto \& Silveira, 2019, p. 36).

O presente estudo consiste em uma pesquisa de natureza básica, documental de caráter descritiva e exploratória, com análise qualitativa que visa descrever dados operacionais do serviço estudado, bem como analisar tais dados a fim de levantar as principais causas locais de superlotação do serviço. O objeto deste estudo trata-se de um do Serviço de urgência e emergência de um Hospital terciário de grande porte do Estado de Sergipe. Este possui atendimento em livre demanda dividido de acordo com o tipo e gravidade.

A pesquisa documental utiliza-se de fontes primárias e/ou secundárias, documentos variados, oriundos de diversos meios, Tal técnica contribui veementemente para ampliação do conhecimento (Sakamoto \& Silveira, 2019).

Conforme Lakatos \& Marconi (2021, p. 186):

Os principais tipos de documentos são:

a) Fontes primárias: dados históricos, bibliográficos e estatísticos; informações, pesquisas e material cartográfico; arquivos oficiais e particulares; registros em geral; documentação pessoal (diários, memórias, autobiografias); correspondência pública ou privada etc.

b) Fontes secundárias: imprensa em geral e obras literárias.

Para a realização desta pesquisa foram utilizadas fontes primárias, pois estamos dispondo de dados ainda não analisados, estudados e interpretados, sem nenhum tratamento de cunho científico, apenas apresentando na íntegra, informações sobre o que se busca. Mas também foram utilizadas fontes secundárias, devido a coleta de informações bibliográficas acerca do assunto abordado.

Para Sakamoto \& Silveira (2019), pode-se dizer que uma pesquisa exploratória tem como finalidade apresentar novos conhecimentos e novos objetos de estudo. Neste contexto, fica claro que tal pesquisa permite elaborar hipóteses e pode servir de base para futuros estudos. Os mesmos autores ainda salientam que, pesquisas descritivas objetivam conhecer um objeto de estudo. Trata-se inegavelmente de adquirir conhecimento através de observação e apresentação detalhada de elementos deste objeto.

Conforme verificado por Gil (2008), a pesquisa qualitativa tem como base que a análise dos dados é dependente do pesquisador. Trata-se inegavelmente de uma pesquisa onde os dados não são quantificáveis, seria um erro, porém, atribuir que dados quantificáveis não são destinados a pesquisa. Assim, reveste-se de particular importância a abordagem quantitativa que faz uso de métricas estatísticas como instrumento de análise, métricas. O método de análise escolhido foi o hipotético-dedutivo pois a pesquisa é realizada em torno de uma hipótese e problema.

O estudo iniciou primeiramente através de uma revisão de literatura nas bases de dados PubMed e Google Acadêmico, que buscou contextualizar o problema da superlotação hospitalar, e como tal situação é analisada e tratada pelo consenso científico. Para tal foram utilizados os descritores "serviço hospitalar de emergência" e "aglomeração". Após revisão, foi realizado levantamento de dados no sistema do Departamento de Informática do Sistema Único de Saúde - DATASUS, utilizando como fonte o Sistema de Informações Hospitalares do SUS (SIHSUS), Sistema de Informação Ambulatorial (SIA) e 
Cadastro Nacional de Estabelecimentos de Saúde (CNES).

O sistema DATASUS foi escolhido como fonte de dados por possuir dados confiáveis e oficiais e de domínio público. Através de suas plataformas, o sistema fornece informações documentais e cruzamento de dados, o que permite analisar da melhor forma a situação da saúde em um determinado território. Com esse tipo de dados foi possível levantar informações importantes sobre o tema e que serviram como fonte para análise.

Os dados coletados foram organizados em planilha do Excel 2016, e posteriormente analisados de acordo modelo conceitual proposto por Asplin et al. (2003), este modelo tem por objetivo facilitar o entendimento da superlotação em serviços de emergência estabelece componentes de análise para as principais causas. Por se tratar de uma pesquisa documental, não foi necessário a submissão deste estudo a um Comitê de ética e pesquisa.

\section{Superlotação de Serviço de Emergência}

Pode-se dizer que a superlotação de um serviço de emergência é quando a quantidade de pacientes presentes no serviço supera a capacidade do mesmo. Neste contexto, Grabois \& Bittencourt (2019) define a superlotação em serviço de emergência como uma consequência de um problema entre demanda e oferta de serviço. O mais tranquilizador, sobretudo, é constatar que tal situação não se restringe há alguns poucos lugares, mas que há, no mundo, uma preocupação com tal problema e as consequências que ele traz.

Conforme verificado por Habib \& Khan (2017), superlotação de serviços de emergência é um problema que vem crescendo gradativamente no mundo. Trata-se inegavelmente de um problema de cunho global, e por isso seria um erro, atribuir que a superlotação de serviços de emergência é apenas um problema do sistema de saúde brasileiro.

Ora, em tese, a superlotação de um serviço de emergência é um problema presente em todo o mundo, conforme explicado acima, mas que conta com particularidades locais de cada região. Caso contrário, todo os processos de superlotação deveriam possuir as mesmas características, causas e consequências. Apesar de global, não se trata de um problema globalmente igual, mas sim um problema presente nos mais diversos países e cada local com suas características. É importante considerar também que apesar de ser uma superlotação do serviço de emergência, a mesma pode ser, na verdade o reflexo ou a consequência de uma superlotação em todo o hospital, o que torna ainda mais preocupante (Salway et al., 2017).

Pode-se dizer que a superlotação de serviços de emergência é um cenário problemático que impede/desregula o seu correto funcionamento e que traz consequências. Tal contexto, deixa claro que a prestação do serviço fica comprometida quando a situação de superlotação está presente, trazendo prejuízos ao serviço e ao atendimento à população (Cameron et al., 2019).

A superlotação nos SEH é reflexo do desequilíbrio entre a demanda de pacientes com condições agudas por serviços de saúde e a baixa oferta de serviços assistenciais. Possivelmente, é a expressão mais visível das dificuldades do sistema de saúde no atendimento das necessidades da população (Grabois \& Bittencourt, 2019, p. 315).

É interessante, aliás, conforme explicado acima, ressaltar que identificar a superlotação em um serviço de emergência é de suma importância, mas há um fato que se sobrepõe a identificação, trata-se da análise e descrição dos fatores causais para que se possa realizar intervenções. É sinal de que há a necessidade de que gestores analisem tanto os cenários de superlotação como também suas causas, isso com o objetivo de impedir as consequências causadas por este problema.

A aglomeração do setor de emergência afeta pacientes individuais, sistemas de saúde e comunidades em geral. As influências negativas da aglomeração na prestação de serviços de saúde resultam em atrasos na prestação de serviços, atendimento de baixa qualidade e ineficiência; todos afetando negativamente os resultados de saúde dos pacientes de emergência, por sua vez (Rasouli et al., 2019, p. 7, tradução nossa). 
É preciso, porém, ir mais além em relação as repercussões causadas pela superlotação de serviços de emergência. É exatamente o que o autor acima deixa claro, a superlotação traz consequências não só ao serviço, mas a todos envolvidos no processo, a exemplo dos pacientes e colaboradores. Por todas essas razões, percebe-se o quão necessário é buscar estudar e analisar a superlotação visando solucionar tal problema.

Fica, portanto, que o que realmente importa, é entender que a superlotação de serviço de emergência é um cenário problemático, reflexo da superlotação hospitalar e que merece esforços de investigação devido as consequências drásticas que desencadeia a todos os envolvidos no processo. Essa, porém, é uma tarefa que demanda interesse, análise e objetivo por parte dos gestores de saúde.

\section{Principais Causas de Superlotação de Serviço de Emergência}

Pode-se dizer que existem três componentes principais dentre as causas de superlotação de serviços de emergência: componentes de entrada (input), componentes de rendimento (throughput) e componentes de saída (output) (Hoot \& Aronsky, 2008). Não é exagero afirmar que o objetivo de compreender as principais causas de superlotação do serviço de emergência é ter base de conhecimento para o desenvolvimento de soluções futuras.

Pode-se dizer que compreender as causas de superlotação é uma ferramenta importante no planejamento para resolução do cenário. Neste contexto, Asplin et al. (2003) desenvolveu um modelo conceitual para ajudar gestores e pesquisadores a compreender as causas de superlotação de serviços de emergência e assim gerar intervenções.

Apesar de ter sido criado há 18 anos atrás, não parece haver razão para que não se utilize tal modelo como base de conhecimento, uma vez que produções científicas mais atuais continuam a utilizar este conceito como base, a exemplo de Bittencourt et al. (2020), Hsu et al. (2019), Morley et al. (2018), Moskop et al. (2019), Salehi et al. (2018). É sinal de que há, uma relevância importante do modelo conceitual no auxílio de pesquisadores em seus trabalhos e na identificação das principais causas estabelecidas.

O conceito de superlotação em um processo assistencial é definido como um desequilíbrio entre o fluxo de pacientes e o dimensionamento do estabelecimento (urgência ou hospital), podendo provocar consequências negativas. É fundamental entender a causa dessa superlotação a fim de remediá-la. (Zgaya \& Hammadi, 2016, p. 100, tradução nossa)

Sendo assim, conhecer as causas de superlotação em um serviço de emergência é um fator importante, por isso nos capítulos seguintes exploraremos melhor estes componentes.

\subsection{Componente de entrada}

O componente de entrada está relacionado ao número de admissões do serviço. Em tese, o componente de entrada do modelo conceitual é toda aquela situação, cenário ou evento que contribuiu para o aumento da demanda de atendimento do serviço. Como bem nos assegura Lindner \& Woitok (2021), pode-se dizer que o componente de entrada é qualquer fator que leve a um aumento de admissões no serviço.

Fatores de entrada: o influxo de pacientes para o departamento de emergência excede a extensão usual em uma quantidade relevante. [...] Em geral, quaisquer fatores que levem a um aumento nas admissões no departamento de emergência podem resultar em situações de superlotação. Vários estudos examinaram as características dos pacientes atendidos em departamentos de emergência e as motivações (Lindner \& Woitok, 2021, p. 2, tradução nossa).

Sendo assim, é possível concluir que as causas referentes aos componentes de entrada de um serviço de emergência 
são fatores que levam ao maior influxo de paciente ao serviço, logo são fatores externos ao serviço.

Conforme verificado por Idil et al. (2018), pode-se dizer que a ida de pacientes não urgentes ao serviço de emergência configura-se como o componente de entrada mais comum no mundo e por consequência uma das principais causas de superlotação de serviço de emergência, por causar uma sobrecarga desnecessária ao serviço. Esse influxo pode ser desencadeado pelas mais diversas motivações da população ou por problemas do sistema de saúde.

\subsection{Componente de rendimento}

O componente de rendimento mostra que há certos fatores causais da superlotação de serviços de emergência que estão atrelados ao cenário interno do serviço. Para Lindner \& Woitok (2021), este componente refere-se a problemas relacionados ao fluxo interno de atendimento do paciente, como problemas de falta de recurso, falta de infraestrutura e que aumentam o tempo de permanência do paciente.

O aumento do tempo de permanência do paciente no serviço é o fator potencial do componente de rendimento e desta forma, contribuem com à superlotação (Asplin et al., 2003).

É importante ressaltar que, conforme explicado acima, existem vários fatores causais que compõem o componente de rendimento, mas, em cima disso, todos possuem como convergência um aumento do tempo de permanência. Como exemplos, podemos citar a demora de um paciente a realizar um exame ou a necessidade de avaliação de um especialista que não se encontra no serviço.

Uma vez que os pacientes atingem a porta, os fatores de rendimento entram em ação, com pacientes sendo tratados em instalações que muitas vezes são incompatíveis em termos de sua capacidade de lidar com seus números e acuidade, como resultado do espaço físico e layout, e os níveis de pessoal que podem oferecer. O acesso a investigações, opiniões de especialistas e outros serviços pode ser limitado (Higginson \& Boyle, 2018, p. 501, tradução nossa).

Por se tratar de um componente interno, faz-se ainda mais necessário um engajamento por parte dos gestores locais, na avaliação dos gargalos de serviço que acabam por ser retentores de pacientes. A resolução desses gargalos, contribuirá com redução da permanência do paciente.

\subsection{Componente de saída}

Podemos conceituar componente de saída como sendo conjunto de fatores que impedem a saída de um paciente do hospital e consequentemente geram a superlotação de um serviço de emergência. Como bem nos assegura Lindner \& Woitok (2021), a indisponibilidade de leitos devido a ocupação de pacientes de longa permanência é um fator causal do componente de saída. Fica claro portanto, que o componente de saída se correlaciona, em certo ponto, com o componente de rendimento e assim podem gerar a superlotação de um serviço de emergência.

É interessante enfatizar que os fatores causais de cada componente se inter-relacionam em certo grau. Mesmo assim, não parece haver razão para que não os abordemos de forma separada, uma vez que são fatores causais distintos. É sinal de que há, enfim, preocupação com o componente de saída apesar deste ser intimamente relacionado os demais.

[...] A disponibilidade de consultas de acompanhamento oportunas no sistema de atenção ambulatorial mais uma vez pode criar problemas de capacidade; no entanto, agora cria gargalos de saída em vez de demandas de entrada para o departamento de emergência. $\mathrm{O}$ tempo gasto pelos provedores de $\mathrm{DE}$ organizando um acompanhamento adequado pode prejudicar a eficiência do atendimento e prolongar o tempo de internação no SEH. [...] (Asplin et al., 2003, p. 3, tradução nossa).

É preciso ressaltar que a saída de um paciente do serviço pode ser adiada devido a diversos fatores, que sejam 
intrínsecos ao paciente seja por questões sociais ou de tratamento, ou a disponibilidade de serviços, e isto infelizmente, eleva o tempo de permanência no serviço, atrasando a alta hospitalar e consequentemente pode levar a superlotação.

Fazendo a junção dos componentes explorados, é possível perceber que quando não há equilíbrio entre os componentes, a superlotação é evento certo. Já que o aumento de influxo somado ao tempo de permanência e atraso na liberação de pacientes é uma equação que resulta em erro.

\section{Resultados e Discussão}

A pesquisa foi realizada por meio da coleta de dados estatísticos e documentais no DATASUS acerca de um hospital público do estado de Sergipe, o Hospital de Urgência de Sergipe Governador João Alves Filho (HUSE). A instituição foi escolhida para esta pesquisa pois é o maior hospital público do estado de Sergipe, consta com disponibilidade de dados estatísticos no departamento, é referência de atendimento em urgência e emergência em todo o estado e possui o cenário de superlotação como um problema de seu serviço.

Os três sistemas pertencentes ao DATASUS e utilizados para coleta de dados referente ao serviço foram o SIHSUS, CNES e o SIA. O SIH fornece dados referentes as internações do serviço de saúde, através dele se buscou obter a quantidade de internações e média de permanência dos pacientes no serviço. No caso do CNES, o sistema nos permitiu obter informações características do serviço, como quantidade de leitos, número de funcionários, etc. Já o SIA nos permite obter informações relacionadas ao atendimento ambulatorial, sendo possível captar a quantidade de atendimentos a nível componente de entrada do serviço.

Para a obtenção dos dados nos sistemas, foi utilizado tanto a plataforma online de consulta disponibilizada pelo DATASUS, o Tabnet, como também o software local, Tabwin, também disponibilizado pelo departamento. Diante das plataformas de consulta foram realizadas aplicações de filtros com o objetivo de obtenção de dados pertinentes a pesquisa. Em um primeiro momento foi obtido a quantidade de leitos presentes na instituição através do CNES bem como a média de permanência e a quantidade de internações através do SIH. Este processo foi realizado utilizando a plataforma Tabnet e em vigência dos seguintes filtros: (i) dados referentes ao HUSE; (ii) dados categorizados por meses, de janeiro de 2019 até dezembro de 2019.

Em um segundo momento, foi realizado a obtenção do número de atendimentos realizado no serviço de urgência através do SIA. Como meio de obter os dados deste sistema, foi-se utilizada o software Tabwin e durante a busca foram utilizados os seguintes filtros: (i) dados referentes ao hospital governador João Alves Filho; (ii) dados categorizados por meses, de janeiro de 2019 até dezembro de 2019; (iii) atendimentos referentes aos procedimentos "atendimento de urgência c/ observação até 24 horas em atenção especializada" e "atendimento de urgência em atenção especializada". Desta forma, tendo como uma pesquisa qualitativa, todos os dados foram catalogados, interpretados pelo autor visando a análise das principais causas do cenário de superlotação do serviço estudado.

Os dados adquiridos tiveram como delimitação serem referentes ao ano de 2019. Tal escolha foi em busca de dados mais atuais possíveis do cenário hospitalar, porém, evitou-se dados pertencentes aos anos de 2020 e 2021, como forma de evitar a presença de viés na pesquisa, uma vez que nesses períodos o sistema de saúde vivenciou uma situação atípica de pandemia e superlotação pela COVID-19.

A primeira questão trata de uma identificação do estado de ocupação que se encontra o serviço de saúde em estudo. Conforme exposto na Tabela 1, percebemos que o hospital durante os meses de 2019 possuiu uma quantidade de internamentos superior à sua quantidade de leitos nos respectivos meses. Chama atenção que, à exceção do mês de janeiro, em todos os meses o volume de internações é mais que o dobro da quantidade de leitos disponíveis. Porém, tal dado não é suficiente para afirmar que há uma superlotação no serviço, uma vez que também se deve considerar o tempo de permanência e a quantidade de saída 
de pacientes do serviço. Desta forma, a média de permanência nos mostra que os pacientes que foram internados permaneceram no serviço por uma média de 11,3 dias e, ao término do período, saem seja por alta, óbito, transferência ou evasão. Sendo assim, fica evidente que o volume excessivo de internações é acompanhado de um tempo de ocupação significativo no hospital. Além dos dados expressos, em entrevista em 2019 o ex-superintendente do HUSE, Darcy Tavares, veio a público expressar preocupação acerca da superlotação do hospital (Sergipe, 2019).

Tabela 1 - Cenário de Superlotação do Hospital de Estudo.

\begin{tabular}{ccccc}
\hline MÊS/ANO & $\begin{array}{c}\text { LEITOS } \\
(\mathrm{n})\end{array}$ & $\begin{array}{c}\text { INTERNAÇÕES } \\
(\mathrm{n})\end{array}$ & $\begin{array}{c}\text { RELAÇÃO LEITOS/INTERNAÇÕES } \\
(\%)\end{array}$ & $\begin{array}{c}\text { MÉDIA DE PERMANÊNCIA } \\
(\text { dias })\end{array}$ \\
\hline JAN/2019 & 567 & 1085 & $191 \%$ & 13 \\
\hline FEV/2019 & 567 & 1312 & $231 \%$ & 12,6 \\
\hline MAR/2019 & 567 & 1180 & $208 \%$ & 12 \\
\hline ABR/2019 & 567 & 1385 & $244 \%$ & 11,5 \\
\hline MAI/2019 & 567 & 1425 & $251 \%$ & 11,2 \\
\hline JUN/2019 & 567 & 1141 & $201 \%$ & 10,5 \\
\hline JUL/2019 & 567 & 1371 & $242 \%$ & 11,2 \\
\hline AGO/2019 & 567 & 1502 & $265 \%$ & 11,6 \\
\hline SET/2019 & 567 & 1763 & $311 \%$ & 11,3 \\
\hline OUT/2019 & 567 & 1750 & $309 \%$ & 10,4 \\
\hline NOV/2019 & 581 & 1277 & $220 \%$ & \\
\hline DEZ/2019 & 581 & 1354 & $233 \%$ & \\
\hline
\end{tabular}

Fonte: Autores, com base em dados do SIHSUS e CNES.

A segunda questão, refere-se a uma análise a respeito das principais causas de superlotação no serviço hospitalar de emergência abordado. "Quais as principais causas de superlotação de um serviço hospitalar de emergência de Sergipe?". De acordo com a Tabela 2, é possível notar que há um grande influxo de pacientes para o serviço. Porém, apesar do grande volume de entrada de pacientes, apenas $14 \%$ dos atendimentos aparentemente se converteram em internações. Na verdade, pode-se dizer que esta porcentagem é menor que a encontrada, uma vez que no total de 16545 internações é contabilizado também as transferências e regulações obtidas no serviço, porém através do DATASUS não é possível identificarmos tais valores. Logo, é possível concluir que as internações provenientes de atendimentos de urgências são menores que a média anual de $14 \%$. Assim, pode-se perceber que $86 \%$, ou mais, atendimentos realizados pela urgência, não ficaram nos serviços, ou seja, não necessitavam do suporte hospitalar, e que deveriam ser absorvidos pela atenção primária e/ou secundária.

Como foi evidenciado por Sergipe (2018), cerca de 90\% dos atendimentos entre janeiro e junho de 2018 do HUSE foram de baixa complexidade e assim não deveriam estar no serviço. Neste contexto, fica claro que não há somente muitas internações como descrito anteriormente, há também um influxo demasiado de pacientes na entrada do serviço, fora do contexto de um hospital terciário. 
Tabela 2 - Atendimentos do Hospital de Estudo.

\begin{tabular}{cccc}
\hline MÊS/ANO & INTERNAMENTOS $(\mathrm{n})$ & ATENDIMENTOS DA URGÊNCIA $(\mathrm{n})$ & TAXA DE CONVERSÃO $(\%)$ \\
\hline JAN/2019 & 1085 & 12008 & $9 \%$ \\
\hline FEV/2019 & 1312 & 9900 & $13 \%$ \\
\hline MAR/2019 & 1180 & 10340 & $11 \%$ \\
\hline ABR/2019 & 1385 & 11774 & $12 \%$ \\
\hline MAI/2019 & 1425 & 11256 & $13 \%$ \\
\hline JUN/2019 & 1141 & 2904 & $39 \%$ \\
\hline JUL/2019 & 1371 & 5750 & $15 \%$ \\
\hline AGO/2019 & 1502 & 10012 & $17 \%$ \\
\hline SET/2019 & 1763 & 10200 & $16 \%$ \\
\hline OUT/2019 & 1750 & 11049 & $13 \%$ \\
\hline NOV/2019 & 1277 & 9927 & $15 \%$ \\
\hline DEZ/2019 & 1354 & 9113 & $14 \%$ \\
\hline TOTAL & 16545 & 114233 & \\
\hline
\end{tabular}

Fonte: Autores, com base em dados do SIA e SIHSUS.

Retomando o que foi exposto na Tabela 1, podemos também evidenciar a presença de uma média de permanência elevada no hospital. Segundo Brasil (2020), o alvo de um hospital é manter esta média abaixo de 5 dias. Considerando os dados de 2019, durante todo o ano a média se manteve acima do ideal. Além disso, em 91\% dos meses a média se apresentou como o dobro do alvo. Neste contexto, em declaração pública, o ex-superintendente do HUSE, Darcy Tavares, referiu ainda que havia também uma falta de insumos e material humano para atender a demanda do hospital (Sergipe, 2019). Assim, fica claro que a média de permanência elevada é uma das consequências da escassez de recursos do serviço, afinal a média também é um indicador de desempenho hospitalar.

Pode-se observar que frente ao modelo proposto por Asplin et al. (2003) para as principais causas de superlotação de um serviço de emergência, o hospital de estudo apresenta fatores causais pertencentes aos 3 componentes do modelo. Em relação ao componente de entrada, observa-se através dos dados expostos anteriormente que o serviço possui um alto influxo de pacientes, porém a grande maioria deste influxo são de casos que não permanecem no serviço, ou seja, casos não urgentes, configurando assim um fator causal deste componente. Em relação aos componentes de rendimento e de saída, se evidenciou através da média de permanência elevada que há a presença de fatores causais nestes componentes. Pode-se dizer que há falta de recursos suficientes para atender a demanda do hospital, evidenciando assim um fator do componente de rendimento. Porém, como dito anteriormente, o componente de saída é intrinsicamente relacionado ao componente de rendimento e desta forma o fator causal de rendimento consequentemente dificulta a saída do paciente do leito, causando assim um problema também do componente de saída.

Dado os componentes apresentados acima como desencadeadores da superlotação do serviço em questão, é notório que este cenário problemático do HUSE não possui uma única causa. De acordo com o que foi exposto por Lin et al. (2019), a superlotação possui causas multifatoriais. Trata-se inegavelmente de um processo complexo, sendo um erro, porém, atribuir uma única causa a tal problema do serviço em questão. Assim, reveste-se de particular importância o incentivo a análises e pesquisas mais aprofundadas acerca de tal problema. 
Vale destacar que para uma análise mais aprofundada do cenário de superlotação no Brasil, se faz necessário a obtenção de mais bases de dados que tragam informações e dados pertinentes a análise do serviço. A exemplo, podemos citar dados como: número de altas, tempo de espera, número de atendimentos diários, etc. Outro destaque é a dificuldade na tabulação dos dados nas plataformas de acesso do DATASUS. Tal limitação foi devida a falta de documentação explicativa acerca do manuseio da plataforma, e acerca dos dados de cada sistema como também a falta de dados nos sistemas em geral. Porém, percebe-se que o problema de dificuldade de acesso já foi identificado pelo departamento, pois durante a pesquisa foi percebido o planejamento de mudança da interface da plataforma, visando uma melhor facilidade no acesso.

\section{Conclusão}

O desenvolvimento da presente pesquisa possibilitou uma análise do cenário de superlotação de um serviço hospitalar de emergência do estado de Sergipe, mas também uma reflexão acerca dos fatores causais que desencadeiam tal problema no serviço através da utilização de recursos didáticos e estatísticos.

De um modo geral, a superlotação do serviço estudado possui suas causas presentes em três pontos no processo de funcionamento do serviço. O primeiro ponto evidenciado foi o grande contingente de pacientes que se direcionam ao serviço buscando atendimento. O segundo ponto é a falta ou atraso em obtenção de insumos e recursos pertinentes ao processo de atendimento dos pacientes internados no serviço. Por último, mas não menos importante, foi observado em conjunto com o ponto anterior a permanência prolongada de pacientes no serviço, assim tal fator dificulta o giro de leitos o que contribui ainda mais para a superlotação.

Concluímos, portanto, que o cenário de superlotação do serviço hospitalar de emergência analisado é um cenário de causa multifatorial. Diante disso, algumas causas já foram identificadas por gestores, porém traçar soluções buscando apenas resolver um componente trarão de início certas melhorias, mas não resolverão o problema.

Dada a importância deste tema, torna-se necessário o investimento e o desenvolvimento de projetos e pesquisas que visem a investigação, análise e coleta de dados para um maior entendimento de todo esse processo complexo que é a superlotação do hospital estudado. Como forma de melhorar a análise institucional seria necessário o cruzamento de dados locais do sistema interno do serviço, ou a correlação com dados colhidos de funcionários e pacientes através de questionários ou pesquisa de campo.

Nesse sentindo, a compreensão dos fatores causais para a superlotação de um serviço permite que gestores e líderes políticos planejem soluções visando a solução de tal problema e consequentemente melhorem a qualidade de assistência prestada a população.

\section{Referências}

Asplin, B. R., Magid, D. J., Rhodes, K. V., Solberg, L. I., Lurie, N., \& Camargo, C. A. (2003). A conceptual model of emergency department crowding. Annals of Emergency Medicine, 42(2), 173-180. https://doi.org/10.1067/mem.2003.302

Bittencourt, R. J., Stevanato, A. de M., Bragança, C. T. N. M., Gottems, L. B. D., \& O’Dwyer, G. (2020). Interventions in overcrowding of emergency departments: An overview of systematic reviews. Revista de Saúde Pública, 54, 66. https://doi.org/10.11606/s1518-8787.2020054002342

Brasil. (2019). Ministério da Saúde. Cadastro Nacional dos Estabelecimentos de Saúde do Brasil-CNES. TabNet Win32 3.0: CNES - Recursos Físicos Hospitalar - Leitos de internação - Sergipe. http://tabnet.datasus.gov.br/cgi/deftohtm.exe?cnes/cnv/leiintse.def

Brasil. (2019). Ministério da Saúde. Sistema de Informações Ambulatoriais do SUS (SIA/SUS).

Brasil. (2019). Ministério da Saúde. Sistema de Informações Hospitalares do SUS (SIH/SUS). TabNet Win32 3.0: Procedimentos hospitalares do SUS - por local de internação - Sergipe. http://tabnet.datasus.gov.br/cgi/deftohtm.exe?sih/cnv/qise.def

Brasil. (2020). PAINEL GERAL: FICHAS TÉCNICAS DOS INDICADORES. Consórcio de Indicadores de Qualidade Hospitalar, 4. https://www.gov.br/ans/pt-br/arquivos/assuntos/prestadores/qualiss-programa-de-qualificacao-dos-prestadores-de-servicos-de-saude-1/1-indicadores-geraisversao-i-publicacao-ans-pdf/view 
Cameron, P., Little, M., Mitra, B., \& Deasy, C. (2019). Textbook of Adult Emergency Medicine E-Book. Elsevier Health Sciences.

Gil, A. C. (2008). Métodos e técnicas de pesquisa social. Atlas.

Grabois, V., \& Bittencourt, R. (2019). Superlotação dos serviços de emergência (p. 315-334). https://doi.org/10.7476/9788575416419.0017

Habib, M. I., \& Khan, K. M. (2017). Overcrowding and possible solutions for a busy paediatric emergency department. JPMA. The Journal of the Pakistan Medical Association, 67(9), 1398-1403.

Higginson, I., \& Boyle, A. (2018). What should we do about crowding in emergency departments? British Journal of Hospital Medicine (London, England: 2005), 79(9), 500-503. https://doi.org/10.12968/hmed.2018.79.9.500

Hoot, N. R., \& Aronsky, D. (2008). Systematic review of emergency department crowding: Causes, effects, and solutions. Annals of Emergency Medicine, 52(2), 126-136. https://doi.org/10.1016/j.annemergmed.2008.03.014

Hsu, C.-M., Liang, L.-L., Chang, Y.-T., \& Juang, W.-C. (2019). Emergency department overcrowding: Quality improvement in a Taiwan Medical Center. Journal of the Formosan Medical Association, 118(1, Part 1), 186-193. https://doi.org/10.1016/j.jfma.2018.03.008

Idil, H., Kilic, T. Y., Toker, İ., Dura Turan, K., \& Yesilaras, M. (2018). Non-urgent adult patients in the emergency department: Causes and patient characteristics. Turkish Journal of Emergency Medicine, 18(2), 71-74. https://doi.org/10.1016/j.tjem.2017.10.002

Lakatos, E. M., \& Marconi, M. de A. (2021). Fundamentos de Metodologia Científica (9a edição). Atlas.

Lin, C.-C., Liang, H.-F., Han, C.-Y., Chen, L.-C., \& Hsieh, C.-L. (2019). Professional resilience among nurses working in an overcrowded emergency department in Taiwan. International Emergency Nursing, 42, 44-50. https://doi.org/10.1016/j.ienj.2018.05.005

Lindner, G., \& Woitok, B. K. (2021). Emergency department overcrowding: Analysis and strategies to manage an international phenomenon. Wiener Klinische Wochenschrift, 133(5-6), 229-233. https://doi.org/10.1007/s00508-019-01596-7

Morley, C., Unwin, M., Peterson, G. M., Stankovich, J., \& Kinsman, L. (2018). Emergency department crowding: A systematic review of causes, consequences and solutions. PloS One, 13(8), e0203316. https://doi.org/10.1371/journal.pone.0203316

Moskop, J. C., Geiderman, J. M., Marshall, K. D., McGreevy, J., Derse, A. R., Bookman, K., McGrath, N., \& Iserson, K. V. (2019). Another Look at the Persistent Moral Problem of Emergency Department Crowding. Annals of Emergency Medicine, 74(3), 357-364. https://doi.org/10.1016/j.annemergmed.2018.11.029

Rasouli, H. R., Aliakbar Esfahani, A., \& Abbasi Farajzadeh, M. (2019). Challenges, consequences, and lessons for way-outs to emergencies at hospitals: A systematic review study. BMC Emergency Medicine, 19(1), 62. https://doi.org/10.1186/s12873-019-0275-9

Sakamoto, C. K., \& Silveira, I. O. (2019). Como fazer projetos de iniciação científica. Pia Sociedade de São Paulo - Editora Paulus.

Salehi, L., Phalpher, P., Valani, R., Meaney, C., Amin, Q., Ferrari, K., \& Mercuri, M. (2018). Emergency department boarding: A descriptive analysis and measurement of impact on outcomes. Canadian Journal of Emergency Medicine, 20(6), 929-937. https://doi.org/10.1017/cem.2018.18

Salway, R., Valenzuela, R., Shoenberger, J., Mallon, W., \& Viccellio, A. (2017). EMERGENCY DEPARTMENT (ED) OVERCROWDING: EVIDENCEBASED ANSWERS TO FREQUENTLY ASKED QUESTIONS. Revista Médica Clínica Las Condes, 28(2), 213-219. https://doi.org/10.1016/j.rmclc.2017.04.008

Sergipe. (2018). 90\% da demanda do Huse deveria ir para as unidades básicas. Saúde. https://www.saude.se.gov.br/90-da-demanda-do-huse-deveria-ir-paraas-unidades-basicas/

Sergipe. (2019). Superintendente do Huse fala sobre situação de superlotação do hospital. Saúde. https://www.saude.se.gov.br/superintendente-do-huse-falasobre-situacao-de-superlotacao-do-hospital/

Zgaya, H., \& Hammadi, S. (2016). Logistics Engineering and Health. Elsevier. 